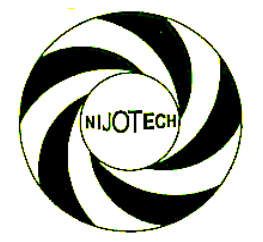

Nigerian Journal of Technology (NIJOTECH)

Vol. 36, No. 2, April 2017, pp. $346-350$

Copyright@ Faculty of Engineering, University of Nigeria, Nsukka,

Print ISSN: 0331-8443, Electronic ISSN: 2467-8821

www.nijotech.com

http://dx.doi.org/10.4314/njt.v36i2.4

\title{
EQUILIBRIUM APPROACH IN THEDERIVATION OF DIFFERENTIAL EQUATIONS FOR HOMOGENEOUS ISOTROPIC MINDLIN PLATES
}

\author{
C. C. Ike* \\ Dept of Civil Engineering, Enugu State University of Science \& TeChnology Enugu, Enugu State, Nigeria \\ E-mail address: ikecc2007@yahoo.com
}

\begin{abstract}
In this paper, the differential equations of Mindlin plates are derived from basic principles by simultaneous satisfaction of the differential equations of equilibrium, the stress-strain laws and the strain-displacement relations for isotropic, homogenous linear elastic materials. Equilibrium method was adopted in the derivation. The Mindlin plate equation was obtained as a system of simultaneous partial differential equations in terms of three displacement variables (parameters) namely $w(x, y, z=0), \theta_{x}(x, y)$ and $\theta_{y}(x, y)$ where $w(x, y, z)$ is the transverse displacement $\theta_{x}$ and $\theta_{y}$ are rotations of the middle surface. It was shown that when $\theta_{x}=-\frac{\partial w}{\partial x}, \theta_{y}=-\frac{\partial w}{\partial x}$ and $k \rightarrow \infty$, where $k$ is the shear correction factor, the Mindlin plate equations reduce to the classical Kirchhoff plate equation which is a biharmonic equation in terms of $w(x, y, z=0)$.
\end{abstract}

Keywords. Mindlin plate, Kirchhoff plate, tranverse displacement, rotations, shear correction factor, biharmonic equation.

\section{INTRODUCTION/LITERATURE REVIEW}

Plates are three dimensional structural members frequently used as fluid containers (circular, elliptical and rectangular plates), building and bridge decks (rectangular and skewed slabs), aircraft wing panels (skewed plates), retaining walls, aerospace panels, and machine components [1 - 4]. Plates can be subjected to in plane loads and transverse loads, and can be simply supported, clamped or free at the edges.

The plate problem belongs to elasticity theory, and is usually to find the distribution of stress fields, strain fields and displacement fields in a given plate under known loading and support conditions. The exact solution is governed by a system of fifteen partial differential equations of equilibrium, material constitutive laws and kinematic equations that are solved subject to the loading conditions and the boundary conditions [5-8].

The basic idea of most plate theories is to reduce the three dimensional problem of plates to two dimensional problems. This reduction of dimension is accomplished by integrating out one of the dimensions, usually the plate's thickness, $Z$; and thus expressing internal stresses using internal stress resultants, $M_{x x}, M_{x y} M_{y y} Q_{x}$ and $Q_{y}$. All plate theories assume a kinematic assumption that the strains can be expanded in the smallest dimension [9, $8,10]$. Accordingly several theories had been formulated to describe the plate problems. Some of these plate theories are Kirchhoff plate theory [3], Mindlin plate theory [4], Reissner plate theory [11, 12], Reddy plate theory[8], Levinson plate theory [13].

In this paper, the Mindlin plate theory for isotropic plates is formulated and derived using the equilibrium method, and presented as a problem of the theory of elasticity.

\section{RESEARCH AIMS AND OBJECTIVES}

The general aim and objective of this study is to derive the Mindlin plate theory from fundamental principles using the equilibrium approach. The specific aims and objectives are

(i) to derive the governing equations of the Mindlin plate theory from fundamental principles of elasticity theory, using the equilibrium approach

(ii) to show the relationship between the Mindlin plate theory and the Kirchhoff-Love plate theory.

\section{DERIVATION OF MINDLIN PLATE EQUATIONS}

The fundamental assumptions are as follows: $[3,4,8,14]$

(i) the state of deformation is described by the transverse displacement in the $z$-direction of the middle surface $w(x, y, z=0)$ and the rotations $\theta_{x}$ and $\theta_{y}$ of the middle surface, where $\theta_{x}$ and $\theta_{y}$ are rotations about the $x$ and $y$ axes of lines normal to the middle surface before deformation [4]. 
(ii) plane cross-sections originally perpendicular to the middle plane of the plate remain plane, but not necessarily orthogonal to the middle surface. Hence according to [14], $\varepsilon_{r} \neq 0, \varepsilon_{x z} \neq 0$.

(iii) the middle surface remains neutral during bending, and is the neutral surface

(iv) the displacement components vary linearly across the thickness

(v) the plate material is isotropic, homogeneous and linear elastic

Let the displacement field be given by

$$
\begin{aligned}
& u(x, y, z)=z \theta_{x}(x, y) \\
& v(x, y, z)=z \theta_{y}(x, y) \\
& w(x, y, z)=w(x, y)
\end{aligned}
$$

In (1) to (3), $u, v, w$ are displacement components in the $x, y, z$ coordinate directions; and $\theta_{x}$ and $\theta_{y}$ are rotations about the $x$ and $y$ axis of lines normal to the middle surface before deformation. The kinematic (straindisplacement) equations for finite strain (smalldisplacement) elasticity problems are thus Equations (4) - (9):

$$
\begin{gathered}
\varepsilon_{x x}=\frac{\partial u}{\partial x} \\
\varepsilon_{y y}=\frac{\partial v}{\partial y} \\
\varepsilon_{z z}=\frac{\partial w}{\partial z} \\
\Upsilon_{x y}=\frac{\partial u}{\partial y}+\frac{\partial v}{\partial x} \\
\Upsilon_{x z}=\frac{\partial u}{\partial z}+\frac{\partial w}{\partial x} \\
\Upsilon_{y z}=\frac{\partial v}{\partial z}+\frac{\partial w}{\partial y}
\end{gathered}
$$

Where $\varepsilon_{x x}, \varepsilon_{y y}, \varepsilon_{z z}$ are normal strains and $\gamma_{x y}, \gamma_{y z}, \gamma_{x z}$ are shear strains. Substitution of Equations (1) - (3) into (4) - (9) gives:

$$
\begin{aligned}
\varepsilon_{x x} & =z \frac{\partial \theta_{x}}{\partial x} \\
\varepsilon_{y y} & =z \frac{\partial \theta_{y}}{\partial y} \\
\varepsilon_{z z} & =0 \\
\Upsilon_{x y} & =z\left(\frac{\partial \theta_{x}}{\partial y}+\frac{\partial \theta_{y}}{\partial x}\right) \\
\Upsilon_{x z} & =\theta_{x}+\frac{\partial w}{\partial x} \\
\Upsilon_{y z} & =\theta_{y}+\frac{\partial w}{\partial x}
\end{aligned}
$$

Plate problems are required to satisfy the stress stain laws, and for two dimensional plane stress problems, we have

$$
\begin{gathered}
\sigma_{x x}=\frac{E}{1-\mu^{2}}\left(\varepsilon_{x x}+\mu \varepsilon_{y y}\right) \\
\sigma_{y y}=\frac{E}{1-\mu^{2}}\left(\varepsilon_{y y}+\mu \varepsilon_{x x}\right)
\end{gathered}
$$

$$
\begin{gathered}
\sigma_{z z}=\mu\left(\sigma_{x x}+\sigma_{y y}\right)\left(\mathrm{NB}: \boldsymbol{\varepsilon}_{y y}=0\right. \\
\left.=\frac{1}{E}\left(\sigma_{z z}-\mu \sigma_{x x}-\mu \sigma_{y y}\right)\right) \\
\tau_{x z}=\frac{E}{2(1+\mu)} \Upsilon_{x z} \\
\tau_{x y}=\frac{E}{2(1+\mu)} \Upsilon_{x y} \\
\tau_{y z}=\frac{E}{2(1+\mu)} \Upsilon_{y z}
\end{gathered}
$$

Here $\sigma_{x x}, \sigma_{y y}$ are normal stresses, $\tau_{x y}$ is the shear stress. These stress-stain laws are expressed in terms of the displacement components by substituting Equations (10) - (15) into Equations (16) - (21) to obtain Equations (22) - (26)

$$
\begin{gathered}
\sigma_{x x}=\frac{E z}{1-\mu^{2}}\left(\frac{\partial \theta_{x}}{\partial x}+\mu \frac{\partial \theta_{y}}{\partial y}\right) \\
\sigma_{y y}=\frac{E z}{1-\mu^{2}}\left(\frac{\partial \theta_{y}}{\partial y}+\mu \frac{\partial \theta_{x}}{\partial x}\right) \\
\tau_{x y}=\frac{E z}{2(1+\mu)}\left(\frac{\partial \theta_{x}}{\partial y}+\frac{\partial \theta_{y}}{\partial x}\right) \\
\tau_{x z}=\frac{E}{2(1+\mu)}\left(\theta_{x}+\frac{\partial w}{\partial x}\right) \\
\tau_{y z}=\frac{E}{2(1+\mu)}\left(\theta_{y}+\frac{\partial w}{\partial y}\right)
\end{gathered}
$$

We wish to eliminate the thickness variable $z$ from the equations, by expressing the internal stresses $\sigma_{x x}, \sigma_{y y}, \sigma_{z z}, \tau_{x z}, \tau_{y z}$ in terms of stress resultants or forces defined as $M_{x x}, M_{y y}, M_{x y}, Q_{x}$ and $Q_{y}$ where $M_{x x}, M_{y y}$ are bending moments, $M_{X y}$, is the twisting moment and $Q_{x}$ and $Q_{y}$ are shear forces.

From statics, the internal stress resultants are obtained as:

$$
\begin{gathered}
M_{x x}=\int_{-h / 2}^{h / 2} \sigma_{x x} z d z \\
M_{y y}=\int_{-h / 2}^{h / 2} \sigma_{y y} z d z \\
M_{x y}=\int_{-h / 2}^{h / 2} \tau_{x y} z d z \\
Q_{x}=\int_{-h / 2}^{h / 2} \tau_{x z} d z \\
Q_{y}=\int_{-h / 2}^{h / 2} \tau_{y z} d z
\end{gathered}
$$

where $\frac{h}{2} \leq z \leq \frac{h}{2}$ and $h$ is the plate thickness.

Using Equations (22) - (26), the internal stress resultants become 


$$
\begin{gathered}
M_{x x}=\int_{-h / 2}^{h / 2} \frac{E z}{1-\mu^{2}}\left(\frac{\partial \theta_{x}}{\partial x}+\mu \frac{\partial \theta_{y}}{\partial y}\right) z d z \\
M_{x x}=\frac{E h^{3}}{12\left(1-\mu^{2}\right)}\left(\frac{\partial \theta_{x}}{\partial x}+\mu \frac{\partial \theta_{y}}{\partial y}\right) \\
=D\left(\frac{\partial \theta_{x}}{\partial x}+\mu \frac{\partial \theta_{y}}{\partial y}\right) \\
M_{y y}=D\left(\frac{\partial \theta_{y}}{\partial y}+\mu \frac{\partial \theta_{x}}{\partial x}\right) \\
M_{x y}=\frac{D(1-\mu)}{2}\left(\frac{\partial \theta_{x}}{\partial y}+\frac{\partial \theta_{y}}{\partial x}\right) \\
Q_{x}=k h G\left(\theta_{x}+\frac{\partial w}{\partial x}\right) \\
Q_{y}=k h G\left(\theta_{y}+\frac{\partial w}{\partial y}\right)
\end{gathered}
$$

where $k$ is the shear correction factor

From the differential equations of equilibrium of an infinitesimal plate element,

$$
\begin{aligned}
& \frac{\partial M_{x x}}{\partial x}+\frac{\partial M_{x y}}{\partial y}=Q_{x} \\
& \frac{\partial M_{x y}}{\partial x}+\frac{\partial M_{y y}}{\partial y}=Q_{y} \\
& \frac{\partial Q_{x}}{\partial x}+\frac{\partial Q_{y}}{\partial y}=-q
\end{aligned}
$$

Substitution of Equations (32) - (37) into Equations (38) - (40), we obtain:

$$
\begin{gathered}
\frac{\partial}{\partial x}\left(D\left(\frac{\partial \theta_{x}}{\partial x}+\mu \frac{\partial \theta_{y}}{\partial y}\right)\right) \\
+\frac{\partial}{\partial y}\left(\frac{D(1-\mu)}{2}\left(\frac{\partial \theta_{x}}{\partial y}+\frac{\partial \theta_{y}}{\partial x}\right)\right) \\
=k G h\left(\theta_{x}+\frac{\partial w}{\partial x}\right) \\
\frac{\partial}{\partial x}\left(\frac{(1-\mu)}{2} D\left(\frac{\partial \theta_{x}}{\partial y}+\frac{\partial \theta_{y}}{\partial x}\right)\right) \\
+\frac{\partial}{\partial x}\left(D\left(\frac{\partial \theta_{y}}{\partial y}+\mu \frac{\partial \theta_{x}}{\partial x}\right)\right) \\
=k G h\left(\theta_{y}+\frac{\partial w}{\partial y}\right) \\
\frac{\partial}{\partial x}\left(k G h\left(\theta_{x}+\frac{\partial w}{\partial x}\right)\right)+\frac{\partial}{\partial y}\left(k G h\left(\theta_{y}+\frac{\partial w}{\partial y}\right)\right) \\
=-q
\end{gathered}
$$

Simplification yields:

$$
\begin{aligned}
D\left(\frac{\partial^{2} \theta_{x}}{\partial x^{2}}+\mu \frac{\partial^{2} \theta_{y}}{d x d y}\right)+\frac{(1-\mu) D}{2}\left(\frac{\partial^{2} \theta_{x}}{\partial y^{2}}+\mu \frac{\partial^{2} \theta_{y}}{d y d x}\right) \\
=k G h\left(\theta_{x}+\frac{\partial w}{\partial x}\right)
\end{aligned}
$$

$$
\begin{aligned}
D\left(\frac{\partial^{2} \theta_{y}}{\partial y^{2}}+\mu \frac{\partial^{2} \theta_{x}}{d_{x} d_{y}}\right)+\frac{(1-\mu) D}{2}\left(\frac{\partial^{2} \theta_{x}}{d_{y} d_{x}}+\mu \frac{\partial^{2} \theta_{y}}{\partial x^{2}}\right) \\
=k G h\left(\theta_{y}+\frac{\partial w}{\partial y}\right)
\end{aligned}
$$

Equations (39) - (40) could be combined to obtain one partial differential equation of equilibrium as follows:

$$
\frac{\partial^{2} M_{x x}}{\partial x^{2}}+2 \frac{\partial^{2} M_{x y}}{\partial x \partial y}+\frac{\partial^{2} M_{y y}}{\partial y^{2}}=-q
$$

The substitution of Equations (33) - (35) in Equation (47) would yield

$$
\begin{gathered}
\frac{D \partial^{2}}{\partial x^{2}}\left(\frac{\partial \theta_{x}}{\partial x}+\mu \frac{\partial \theta_{y}}{d_{y}}\right)+\mu \frac{D(1-\mu)}{2} \frac{\partial^{2}}{\partial x \partial y}\left(\frac{\partial \theta_{x}}{\partial y}+\frac{\partial \theta_{y}}{\partial y}\right) \\
+\frac{D \partial^{2}}{\partial y^{2}}\left(\frac{\partial \theta_{y}}{\partial y}+\mu \frac{\partial \theta_{x}}{d_{x}}\right)=-q
\end{gathered}
$$

Simplification yields:

$$
\nabla^{2}\left(\frac{\partial \theta_{x}}{\partial x}+\mu \frac{\partial \theta_{y}}{\partial y}\right)=-\frac{q}{D}
$$

where $\nabla^{2}$ is the Laplacian operator.

The governing equation of isotropic homogeneous Mindlin plates are thus obtained as a system of differential equations in terms of three unknown displacement parameters $w, \theta_{x}$ and $\theta_{x}$. They are Equations (44), (45), (46) and (49).

\section{RELATIONSHIP BETWEEN THE MINDLIN PLATE THEORY AND THE CLASSICAL KIRCHHOFF PLATE THEORY}

In the classical Kirchhoff plate theory, the shear strains $\gamma_{x z}$ and $\gamma_{y z}$ are disregarded; and assumed to be equal to zero, respectively. Then Equations (14) and (15) would yield

$$
\begin{aligned}
& \gamma_{x z}=\theta_{x}+\frac{\partial w}{\partial x}=0 \\
& \gamma_{y z}=\theta_{y}+\frac{\partial w}{\partial y}=0
\end{aligned}
$$

Hence,

$$
\begin{aligned}
& \theta_{x}=-\frac{\partial w}{\partial x} \\
& \theta_{y}=-\frac{\partial w}{\partial y}
\end{aligned}
$$

The internal bending moment resultants Equations (33) - (35) would then become for the Kirchhoff plate theory:

$$
\begin{gathered}
M_{x x}=D\left(\frac{\partial}{\partial x}\left(-\frac{\partial w}{\partial x}\right)+\mu \frac{\partial}{\partial y}\left(-\frac{\partial w}{\partial y}\right)\right) \\
M_{x x}=-D\left(\frac{\partial^{2} w}{\partial x^{2}}+\mu \frac{\partial^{2} w}{\partial y^{2}}\right) \\
M_{y y}=-D\left(\frac{\partial^{2} w}{\partial y^{2}}+\mu \frac{\partial^{2} w}{\partial x^{2}}\right) \\
M_{x y}=-D(1-\mu) \frac{\partial^{2} w}{\partial x \partial y}
\end{gathered}
$$

The shear force resultants are

$$
Q_{x}=0
$$




$$
Q_{y}=0
$$

The equation of equilibrium of Mindlin plate when shear strains are disregarded becomes:

$$
\begin{aligned}
\nabla^{2}\left(\frac{\partial \theta_{x}}{\partial x}+\frac{\partial \theta_{y}}{\partial y}\right) & =\nabla^{2}\left(\frac{\partial}{\partial x}\left(-\frac{\partial w}{\partial x}\right)+\frac{\partial}{\partial y}\left(-\frac{\partial w}{\partial x}\right)\right) \\
& =-\frac{q}{D} \\
-\nabla^{2} \nabla^{2} w & =-\frac{q}{D} \text { or } \nabla^{2} \nabla^{2} w=\frac{q}{D}=\nabla^{4} w
\end{aligned}
$$

where $\nabla^{2}$ is the Laplacian operator while $\nabla^{4}=\nabla^{2} \nabla^{2}$ is the biharmonic operator.

Equation (61) is the governing partial differential equation of equilibrium for Kirchhoff plates. The Mindlin plate governing equation thus reduces to the classical Kirchhoff plate equation when. $\varphi_{x}=\frac{\partial w}{\partial x}$ and $\varphi_{y}=-\frac{\partial w}{\partial y}$

\section{DISCUSSIONS AND CONCLUSIONS}

The Mindlin plate theory has been derived for isotropic, homogeneous linear elastic plates using the equilibrium method, taking into consideration the shear strain, and hence the shear stress across the plate thickness. However, the shear strain is assumed to be constant across the plate thickness, violating the predictions of the theory of elasticity since the shear stress is known to vary parabolically over the plate thickness. A shear correction factor $k$, is introduced to ensure that the correct amount of internal energy is predicted by the Mindlin plate theory. The shear correction factor merely yields a resultant shear stress that agrees with the predications of elasticity theory, but the distribution of shear stress and shear strain violate the theory of elasticity solutions. The transverse shear stresses are found to be constant over the plate thickness, contradicting the shear free boundary condition on the plate surfaces.

The differential equations for Mindlin plates have been derived in this paper from fundamental principles using the equilibrium method. The equations were derived using the requirements of the differential equations of equilibrium for an infinitesimal element of the plate, the material constitutive laws, and the strain-displacement relations for small-displacement elasticity problems. This three sets of relations were assumed to be satisfied simultaneously for any differential element of the plate. The deformation field was assumed, in line with Mindlin's plate to be defined by three displacement components, namely the transverse displacement $w$, and the rotations $\theta_{x}$ and $\theta_{y}$ relaxing the orthogonality requirements of plane cross sections to the middle (neutral) surface, by enforcing $\varepsilon_{x y} \neq 0$ and $\varepsilon_{x z} \neq 0$ shear strains were accounted for in the derivation, yielding stress-strain laws given by Equations (16) - (21). The material constitutive laws expressed in terms of the three displacement fields were obtained as Equations (22) - (26). The internal stress resultants were obtained as Equations (33) - (37). Differential equations of equilibrium expressed in terms of the internal stress resultants were then used to obtain the governing partial differential equations of equilibrium of Mindlin plates as Equations (44) - (46) and (49). The governing equations of Mindlin plates are a system of partial differential equations in terms of three unknown displacement parameters, namely $w, \theta_{x}$ and $\theta_{y}$.

Mindlin theory is a two dimensional plate theory and cannot yield an exact solution to plate problems since plate problems are three dimensional. The major merits of the Mindlin plate theory are that the theory takes consideration of shear effects, and the theory simplifies to the classical Kirchhoff plate theory by setting $\theta_{x}=$ $-\frac{\partial w}{\partial x} \theta_{y}=-\frac{\partial w}{\partial y}$, and $k \rightarrow \infty$. The shear correction factor introduced to account for the non-uniformity of the shear strain on the cross-section is dependent on the shape of the cross-section, and the shear stress distribution. Reissner suggests that $\mathrm{k}=5 / 6$.

\section{REFERENCES}

[1] Timoshenko S.P. and Woinowsky-Krieger . Theory of Plates and Shells. Second Edition McGraw Hill Publishing Company, p.2.1987

[2] Chen C. E. Application of the Perturbation method to the small and large deflection problems of clamped plates. Master of Applied Science thesis University of Ottawa, Ottawa, Canada March 1970.

[3] Chandrashekhera K. Theory of Plates. University Press (India) limited Hyderabad. 2001.

[4] Mindlin R. D. Influence of rotatory inertia and shear on flexural motions of isotropic, elastic plates. Journal of Applied Mechanics, pages 31-38. 1951.

[5] Nishawala V.V. A study of large Deflection Beams and Plates MSc Thesis Graduate School, New Brunswick, Rutgers, the state University of New Jersey. 2011.

[6] Golterman P. Reinforced Concrete Slabs-Analysis and Design An electronic textbook for basic concrete courses Department of Civil Engineering \&TU, Civil Engineering, Technical University of Denmark 2013.

[7] Kumar S. K. Analysis of Composite Plates using Element Free Galerkin Method Master of Technology (Structural Engineering) Thesis National Institute of Technology, Rourkela June 2013.

[8] Reddy J. N. Theory and Analysis of Elastic Plates and Shells. Second Edition CRC Press, Taylor and Francis Group USA. 2007 
[9] Szilard P. Theories and Applications of Plate Analysis; Classical, Numerical and Engineering Methods. John Wiley and Sons Inc., New Jersey. 2004.

[10] Bletzinger Kai-Une Theory of Plates Part II. Plates in Bending Lecture Notes Winter Semester 2012/2013 Lehnstahl fir Static Technische Universitat Munchen October, 2008 https//www.swibd.com/doc/112345132/theoryof plates-part 22008.

[11] Reissner E. . The effect of transverse shear deformation on the bending of elastic plates. Journal of Applied Mechanics, page (A) 69-77, June, 1945.
[12] Speare P.R.S Shear Deformation in Elastic Beams and Plates PhD Thesis University of London, April 1975

[13] Wang C. M., Reddy J. N. and Lee K.H. Shear Deformable Beems and Plates: Relationships with Classical Solutions. Elsevier Service Ltd Amsterdam Oxford U.K. 2000.

[14] Durban D., Givoli D., and Simmouds J.G. Editors . Solid Mechanics and its Applications Advances in the Mechanics of Plates and Shells: The Avionoam Libai Anniversary Volume, Klumer Academic Publishers Dordrecht, the Netherlands. 2001. 